IdeAs

Idées d'Amériques

12 | 2018

Le tourisme dans les Amériques

\title{
Pour les Afro-Américains, Donald Trump est-il un président républicain comme un autre?
}

Lisa Veroni-Paccher

\section{OpenEdition}

1 Journals

Édition électronique

URL : https://journals.openedition.org/ideas/4418

DOI : 10.4000 /ideas 4418

ISSN : 1950-5701

Éditeur

Institut des Amériques

Référence électronique

Lisa Veroni-Paccher, «Pour les Afro-Américains, Donald Trump est-il un président républicain comme un autre? », IdeAs [En ligne], 12 | 2018, mis en ligne le 14 novembre 2018, consulté le 19 octobre 2022. URL : http://journals.openedition.org/ideas/4418; DOI : https://doi.org/10.4000/ideas.4418

Ce document a été généré automatiquement le 19 octobre 2022

\section{(c) (i) () $९$}

Creative Commons - Attribution - Pas d'Utilisation Commerciale - Pas de Modification 4.0 International - CC BY-NC-ND 4.0

https://creativecommons.org/licenses/by-nc-nd/4.0/ 


\title{
Pour les Afro-Américains, Donald Trump est-il un président républicain comme un autre?
}

\author{
Lisa Veroni-Paccher
}

1 Lors de sa campagne électorale, Donald Trump s'est distingué des autres candidats par son discours raciste et sa personnalité incontrôlable. Alors qu'il a réussi à séduire une partie de l'électorat blanc, il n'a obtenu que $8 \%$ des voix noires. Pourtant, le candidat a promis d'obtenir $95 \%$ du vote noir en 2020 et s'est donc engagé à offrir un New Deal, for Black America, une «nouvelle donne » aux Afro-Américains, en clin d'œil aux politiques publiques de Roosevelt. Néanmoins, la situation économique, sociale et politique des Afro-Américains est aujourd'hui peu réjouissante. Une fois élu, Trump a-t-il, comme Obama avant lui, tenté de lutter contre les inégalités ethnoraciales ? Peut-on retracer des politiques concrètes du gouvernement actuel en faveur de ce groupe ethnoracial? Par ailleurs, Donald Trump se distingue-t-il des autres présidents républicains de la fin du XXème siècle dans sa relation avec les Afro-Américains, citoyens comme élus?

\section{Nous, le peuple, en vue de former une union plus parfaite}

2 Si, à l'inverse de Trump, Barack Obama a été plébiscité par les électeurs noirs, avec son discours d'inclusion et d'union nationale en référence au préambule de la Constitution, en 2016, les inégalités ethnoraciales allaient toujours croissant et ce depuis les années 1970. A la fin de son deuxième mandat:

3 le revenu médian des foyers afro-américains était le moins élevé de tous les groupes ethnoraciaux et demeurait largement en deçà du revenu médian national. Contrairement à tous les autres, les Afro-Américains n'avaient toujours pas retrouvé le niveau de vie d'avant la Récession de 2001, puis avaient été les plus durement touchés par la Grande Récession de 2008.

- le taux de chômage et de pauvreté des Afro-Américains dépassait la moyenne nationale et représentait le double de celui des Américains blancs. 
- les Afro-Américains bénéficiaient le moins souvent d'une couverture sociale et demeuraient le groupe dont les problèmes de santé affectent le plus la qualité de vie.

- le système scolaire restait fortement ségrégué, ce qui a un impact négatif sur leur niveau scolaire.

- alors qu'ils ne représentent que 13,4\% de la population, les Afro-Américains constituent le tiers de la population carcérale. En 2016, ils étaient aussi plus souvent victimes de violences policières et de profilage racial, ainsi que du stop-and-frisk (interpellation et fouille) qui viole leurs droits constitutionnels.

Obama a-t-il tenté de remédier aux inégalités ethnoraciales? Les politiques publiques mises en place entre 2008 et 2016 pour les atténuer n'ont pas toujours été conçues pour cibler les Afro-Américains ou les groupes ethnoraciaux de manière spécifique. Obama a toujours souhaité que sa politique transcende la question ethnoraciale. La transcendance de la question ethnoraciale était avant tout une stratégie politique - une stratégie postraciale -; il était important pour l'ancien président de ne pas être considéré comme le président des Afro-Américains. Son administration a donc mis en place une réforme universaliste de l'assurance maladie en 2010 afin de limiter les conséquences de la pauvreté sur la santé: le pourcentage d'Afro-Américains non retraités sans couverture sociale est passé $18,9 \%$ à $11 \%$ entre 2013 et 2016 . Ce gouvernement a investi des milliards de dollars dans le redressement économique du pays suite à la Grande Récession pour relancer l'emploi et protéger les Américains souhaitant contracter un emprunt, et notamment les Afro-Américains, victimes les plus fréquentes des subprimes. Ainsi, le taux de chômage des Afro-Américains qui s'était élevé à 16,8\% en mars 2010 est retombé à 7,9\% en décembre 2016. D'autres mesures ont un caractère plus ciblé, car l'administration Obama a tout de même estimé qu'il fallait combiner des mesures universalistes et des mesures destinées aux Afro-Américains : la loi de régulation des marchés financiers et de protection des consommateurs DoddFrank de 2010 contient des références explicites à l'identité ethnoraciale, que les services financiers doivent prendre en considération afin de protéger les consommateurs de tout risque financier. Dans le domaine de l'éducation, l'administration Obama a concentré ses efforts sur les groupes marginalisés mais n'a statistiquement pas réussi à ralentir le phénomène de ségrégation scolaire. De nombreuses directives ministérielles ont aussi été adoptées afin de prendre en compte l'identité ethnoraciale des étudiants comme critère d'admission dans les établissements universitaires, encourageant par ce biais l'Affirmative Action. Dans le domaine de la justice, suite à un rapport officiel de 2016 décrivant les prisons privées comme violentes et inefficaces, et dont l'offre de réinsertion demeure trop limitée pour la population carcérale, encore plus disproportionnellement jeune et noire que dans les prisons publiques, l'administration Obama a choisi de ne progressivement plus avoir recours à ces institutions carcérales. Le ministère de la Justice a en outre mené plus d'une vingtaine d'enquêtes dans des services de police, à Baltimore, Ferguson et Chicago notamment, afin d'évaluer l'ampleur des violences policières contre les AfroAméricains et de renforcer la supervision des services de proximité. Malgré une volonté de lutter contre les inégalités, la réussite du gouvernement dans ce domaine demeure limitée, et concerne principalement le taux d'emploi des Afro-Américains. Cette incapacité à endiguer les disparités ethnoraciales contraste avec l'optimisme rhétorique d'Obama et sa vision d'une Amérique « plus parfaite ». 
"Qu'avez-vous à perdre? Vous vivez dans la pauvreté, vos écoles ne sont pas bonnes, vous n'avez pas de boulot, 58\% de vos jeunes sont au chômage ».

Donald Trump, discours de Dimondale, Michigan, 19 août 2016

Alors que ces disparités semblent faire partie intégrante du système institutionnel, Trump a-t-il à son tour tenté de les endiguer une fois élu ? Les mesures de la «nouvelle donne " devaient comprendre, entre autres, l'abandon de la loi Dodd-Frank, l'investissement dans de nouvelles infrastructures et la création d'emplois, spécialement dans les centres-villes qui concentrent les populations noires et défavorisées, la subvention d'écoles privées sous contrat et le financement des institutions universitaires historiquement afro-américaines (Trump/Pence Make American Great Again, 26 octobre 2016). Déjà, les budgets des deux premières années de la présidence n'ont pas consacré les sommes promises dans ces investissements. Le Congressional Black Caucus ( $\mathrm{CBC}$ ), la coalition afro-américaine au Congrès, a affirmé dans une lettre de mars 2017 intitulée "Nous avons beaucoup à perdre " (Congressional Black Caucus, 13 mars 2017) que de telles propositions ne tiennent pas compte des problèmes fondamentaux qui fragilisent les Afro-Américains dans leur majorité. Par la suite, la coalition a vivement critiqué le refus du président de condamner les manifestants d'extrême droite de Charlottesville et son choix de qualificatif des pays d'Afrique comme « pays de merde » et réclame aujourd'hui sa destitution.

Que reproche concrètement le $\mathrm{CBC}$ au président en dehors de la virulence de sa rhétorique de division raciale ? Dans quelle mesure la politique de l'administration Trump porte-elle préjudice aux Afro-Américains?

Pour relancer l'économie, le Congrès a en mai 2018 assoupli les règles de régulation bancaire qui protégeaient les consommateurs depuis 2010. Par conséquent, les AfroAméricains ne sont aujourd'hui pas mieux protégés des effets d'une potentielle crise économique qu'avant le passage de la loi Dodd Frank. Dans son discours de l'Union de 2018, Trump s'est glorifié d'être responsable de la baisse du taux de chômage des AfroAméricains. Non seulement ce taux décline de manière stable depuis 2010 mais Trump n'a pas créé les emplois promis en 2016, puisque la création d'emplois en 2017 fut plus faible que l'année précédente. Trump s'était aussi engagé à démanteler l'Obamacare, mais il n'a réussi à le faire que de manière partielle. Le taux de personnes sans protection sociale était de $10,9 \%$ à la fin du mandat d'obama; il est passé à $12,3 \%$ en 2018. Dans le domaine de l'éducation, l'administration Trump tente aussi de démanteler les lois passées par le gouvernement précédent. S'il n'y a pas de consensus sur l'efficacité de ces dernières, statistiquement, la ségrégation scolaire empire, surtout dans les écoles sous contrat soutenues par l'administration actuelle. De plus, les directives visant à maintenir la prise en compte du facteur ethnoracial dans les universités ont été révoquées en juillet 2018. Enfin, les fonds alloués aux institutions universitaires historiquement noires ont diminué. Rien dans les mesures passées ne montre une volonté de réduire les inégalités ethnoraciales. Dans le domaine de la justice, l'administration Trump a mis fin en février 2017 au programme visant à ne plus recourir aux institutions carcérales privées. Alors que depuis 2012, le nombre d'incarcérations dans ces prisons était en baisse notable, depuis 2017, la tendance s'est inversée: les conditions de vie des jeunes Afro-Américains, majoritaires dans ces prisons, se sont donc détériorées. Les mesures gouvernementales mises en place depuis 
deux ans, présentées comme étant en faveur des Afro-Américains, sont en réalité un rejet des mesures prises par le gouvernement précédent et une volonté de détruire le fragile héritage d'Obama. L'administration actuelle, en défendant une politique d'exclusion des Afro-Américains, divise la nation et prend une part active dans le maintien des inégalités. Trump est-il le seul responsable?

Rendre sa grandeur à l'Amérique

Trump avait promis une nouvelle donne aux Afro-Américains; les Etats républicains, surtout ceux du Sud, n'ont, quant à eux, pas attendu pour proposer une «nouvelle donne " par le biais d'une redistribution des forces électorales par laquelle les électeurs noirs ne ressortent pas gagnants. Dès 2010, une quinzaine d'Etats républicains, particulièrement ceux du Sud, ont créé des restrictions visant principalement les AfroAméricains et les Hispaniques défavorisés, comme la nécessité de présenter une pièce d'identité avec photo ou l'absence de possibilité de voter par correspondance, en contradiction avec la loi sur le droit de vote de 1965. La décision Shelby County v. Holder a, en 2013, assoupli cette même loi en mettant fin au régime de tutelle des Etats du Sud et des collectivités locales anciennement ségrégationnistes. Depuis, des milliers de bureaux de vote ont été fermés dans les comtés majoritairement afro-américains. Le durcissement des conditions de vote dans les Etats républicains avait déjà conduit en 2016 au déclin du taux de participation et du nombre d'électeurs noirs potentiel le plus important depuis vingt ans. Cette tendance ne peut que s'accélérer suite à la décision de la Cour en juin 2018 de considérer comme conforme à la Constitution le plan des Etats d'expurger leurs listes électorales des électeurs occasionnels, plus souvent afroaméricains ou hispaniques et pauvres. Après la nomination de Jeff Sessions, le ministère de la Justice a en parallèle mis un terme aux enquêtes visant les services de police pour violences ; Trump lui-même a incité la police à utiliser le stop and frisk, signalant par là le retour d'une politique de renforcement de la lutte contre la criminalité qui menace les droits constitutionnels des populations les plus démunies. $\mathrm{Si}$ Trump préoccupe parfois le Parti républicain par sa personnalité provocatrice, la ligne politique de l'ancien magnat de l'immobilier et celle de son administration semblent être, dans le domaine de la lutte contre les inégalités, en conformité avec celle du Parti. Cette stratégie républicaine arrive ainsi à son point culminant, se consacrant à élargir toujours davantage l'éventail des possibilités de démobilisation électorale des AfroAméricains, qui restent toujours captifs du Parti démocrate. Cette stratégie d'exclusion renforce aujourd'hui le réalignement politique selon un axe ethnoracial. Elle s'accompagne désormais d'une rhétorique offensante décomplexée qui serait devenue politiquement acceptable.

Trump n'est pourtant pas le premier président républicain à instrumentaliser une telle rhétorique raciste. Comme Reagan avant lui, il a promis - c'est son slogan de campagne - de "rendre sa grandeur à l'Amérique", s'appuyant sur cette vulnérabilité que ressentent de nombreux Américains blancs suite à l'élection d'un président noir. Ce sentiment est renforcé par les crises économiques qui ont secoué la nation et par la transformation du paysage démographique, toujours plus multiracial. En lançant la campagne de stigmatisation des mères célibataires, souvent afro-américaines, Reagan aimait aussi ponctuer ses discours de références aux "Reines de l'assistance ». Le candidat Bush père lui se référait au criminel afro-américain Willie Horton pour alimenter la peur de l'autre et rendre responsable son prédécesseur démocrate de 
l'insécurité. D'autres Républicains vont jusqu'à exiger de "reprendre l'Amérique ». Trump va plus loin: la différence entre le président actuel et ses prédécesseurs républicains réside dans ce discours incitant à la haine raciale de manière plus ouverte qu'avant, affirmant que les athlètes afro-américains sont stupides et méritent de se faire insulter, ou que John Lewis, un des leaders du Mouvement des droits civiques et membre du CBC, n'est qu'un «beau parleur ». Ce refus de respecter les codes établis du discours politique a fait son succès. Son intolérance incite donc un nombre croissant d'Américains à exprimer leurs préjugés et leur rejet de l'autre ouvertement mais permet aussi à ceux qui n'adhérent pas à ce discours de prendre pleinement conscience des multiples facettes du racisme. En effet, avec Trump, le racisme qui, aux Etats-Unis, est historiquement systémique, institutionnel et structurel, redevient aussi intentionnel. Son arrivée au pouvoir est-elle la preuve qu'il n'y a plus de prix à payer pour les hommes politiques qui font des remarques racistes (ou sexistes) explicites (Schaffner B., Mac Williams M., Nteta T., 2018)? Si c'est le cas, il est probable que sa stratégie électorale soit choisie par davantage de candidats dans le futur, rendant plus aisé le déni de la persistance des disparités ethnoraciales et modelant une Amérique toujours plus polarisée.

Donald Trump demeure le produit de plus de cinquante ans de stratégie politique d'exclusion des Afro-Américains. Comme Goldwater, Nixon et Reagan avant lui, Trump poursuit la fabrication délibérée d'une identité nationale mythique en proposant une ré-historicisation de l'Amérique : une nation blanche, masculine et conservatrice, dite libre. Au cœur du système institutionnel, malgré son programme à contre-courant, le Parti démocrate n'a pas réussi à convaincre qu'il pouvait déconstruire cette Amérique mythique, en adaptant jusqu'à la campagne d'Hillary Clinton une stratégie visant à valoriser les identités ethnoraciales. Pourtant, les actes de résistance se multiplient : les femmes américaines, et notamment les femmes noires, font campagne pour les élections de mi-mandat et pour celles de 2020. Si les Républicains ont deux fois moins de candidats afro-américains qu'en 1994 et 2000, les Démocrates ont aujourd'hui au moins 62 candidats afro-américains pour la Chambre des représentants. Black Voters Matter et la campagne Unity ' 18 , ont permis à Stacey Abrams de remporter les primaires de son parti ; elle pourrait devenir la première femme noire gouverneur de la Géorgie. Les élections de mi-mandat testent à nouveau la résilience des institutions américaines, dont la force devrait être le système des freins et contrepoids entre les pouvoirs exécutif, législatif et judiciaire. Ces institutions sont marquées par un déficit démocratique sans précédent, que la mobilisation électorale derrière un candidat comme Bernie Sanders avait cherché à combler. A l'extérieur de ce système, des mouvements émergent et recherchent l'expression démocratique, comme Black Lives Matter. Ces différentes formes de mobilisation seront-elles suffisantes pour préserver les fragiles principes de démocratie et d'égalité dont les Etats-Unis se targuent d'être les garants? 


\section{BIBLIOGRAPHIE}

Congressional Black Caucus. « We Have a Lot to Lose: Solutions to Advance Black Families in the 21st Century ». 13 mars 2017. Washington D.C. Disponible en ligne : https://cbc.house.gov/ wehavealottolose/. Page consultée le 4 avril 2018.

Schaffner, Brian, Matthew Mac Williams, Tatishe Nteta, "Explaining White Polarization in the 2016 Vote for President : The Sobering Role of Racism and Sexism" Political Science Quarterly, vol. 133, n²1, Printemps 2018, p.4-33.

Trump/Pence Make American Great Again. « Trump's New Deal for Black America with a Plan for Urban Renewal ». 26 Octobre 2016. Disponible en ligne : https://assets.donadljtrump.com/ Plan_For_Urban_Renewal.pdf. Page consultée le 5 juin 2018.

\section{AUTEUR}

\section{LISA VERONI-PACCHER}

Maître de conférences, Civilisation américaine, Université Bordeaux Montaigne 